\title{
Odd-Even Effect in the Formation and Extraction Performance of Ionic-Liquid-Based Aqueous Biphasic Systems
}

\author{
Diana C. V. Belchior, Mafalda R. Almeida, Tânia E. Sintra, Sónia P. M. Ventura, ${ }^{\circledR}$ Iola F. Duarte, \\ and Mara G. Freire*0
}

CICECO-Aveiro Institute of Materials, Department of Chemistry, University of Aveiro, 3810-193 Aveiro, Portugal

\section{Supporting Information}

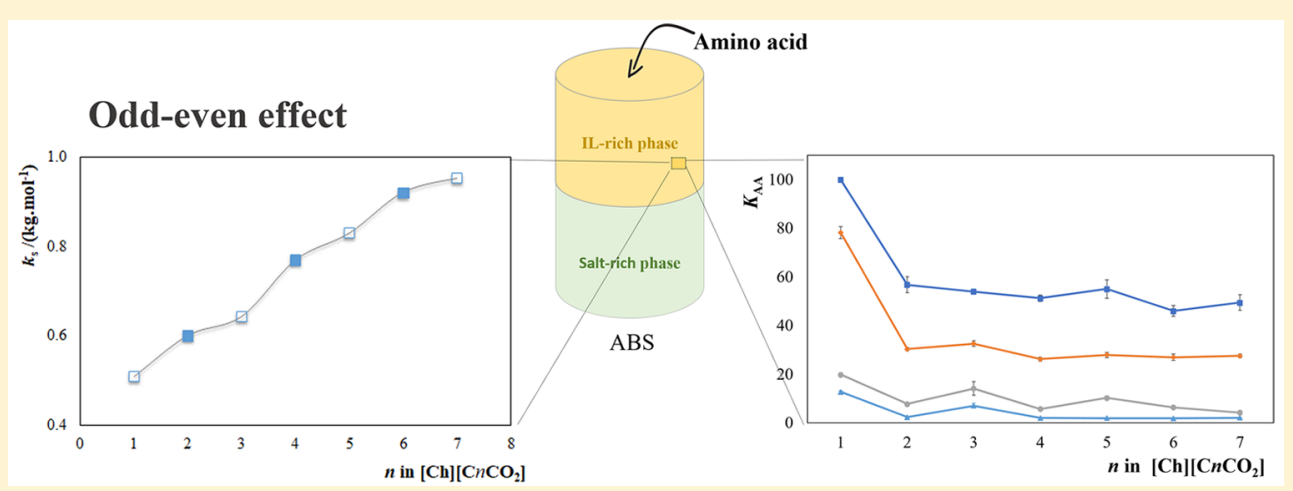

ABSTRACT: Aqueous biphasic systems constituted by ionic liquids (IL-based ABSs) are a target of investigation in the separation of high-value biomolecules. However, identification of the molecular-level mechanisms ruling the two-phase formation and extraction performance of these systems is crucial to the successful design of effective separation processes. In this work, IL-based $\mathrm{ABSs}$ formed by $\mathrm{K}_{2} \mathrm{HPO}_{4}$ and cholinium carboxylate ILs $\left([\mathrm{Ch}]\left[\mathrm{C}_{n} \mathrm{CO}_{2}\right]\right.$ with $n=1-7$, comprising anions with odd and even alkyl chain lengths) were investigated. The corresponding ternary phase diagrams, including binodal curves, tie-lines, tie-line lengths, and critical points, as well as the Setschenow salting-out coefficients $\left(k_{\mathrm{s}}\right)$, which quantitatively describe the two-phase formation ability, were determined at $298 \mathrm{~K}$. The extraction capability of these systems was then evaluated for four amino acids (L-tryptophan, L-phenylalanine, L-tyrosine, and L-3,4-dihydroxyphenylalanine/L-dopa). It was found that ILs composed of anions with even alkyl chains display slightly higher $k_{\mathrm{s}}$ values, meaning that these ILs are more easily salted out or more easily phase-separated to form ABSs, whereas ABSs formed by ILs with anions comprising odd alkyl chains lead to slightly higher partition coefficients of amino acids. Beyond the neat IL odd-even effect resulting from their nanostructuration, being this a well-known phenomenon occurring in a series of their thermophysical properties, the existence of an odd-even effect displayed by the IL anion aliphatic moiety in aqueous solution is shown here, visible in both the two-phase formation ability and extraction performance of ABSs. These findings contribute to elucidate of the molecular-level mechanisms governing ABS formation and partitioning of biomolecules, ultimately contributing to the design of proficient separation platforms.

\section{INTRODUCTION}

Separation and purification steps within biotechnological processes are still major challenges because of difficulties in purifying and recovering the target compounds from the original complex media in which they are produced. ${ }^{1}$ Furthermore, these should be cost-effective and should be able to keep the products' biological activity. Research on alternative separation techniques has been carried out with aqueous biphasic systems (ABSs), which correspond to liquid-liquid extraction systems formed by water and two solutes (e.g., a polymer and a salt, two polymers, two salts, etc.) that undergo phase separation above the defined concentrations. ${ }^{2-5}$ In ABSs, both phases are majorly constituted of water, meaning that such media may be compatible with biologically active molecules. ${ }^{2}$

In addition to more conventional ABSs formed by polymers, in the past decade, a substantial interest has been placed on ionic liquid (IL)-based ABSs, initially demonstrated to be formed by adding ILs to inorganic salt aqueous solutions. ${ }^{6}$ ILs are poorly coordinated organic salts and hence may be liquid at or close to room temperature. Besides other relevant properties, the major advantage associated with IL-based ABSs relies on these compounds' designer ability, resulting in a virtually endless opportunity of tuning the ILs' chemical structures for specific applications. ${ }^{7}$ As a result, IL-based ABSs have been successfully applied in the separation of a wide number of biomolecules, such as amino acids, proteins, antioxidants, among others. ${ }^{8}$ Still, most of the ILs studied to date are imidazolium-based, most of the

Received: February 1, 2019

Revised: April 13, 2019

Accepted: April 18, 2019

Published: April 18, 2019 


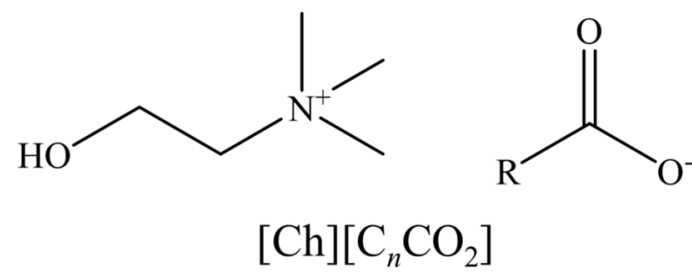

$(n=1,2,3,4,5,6,7)$
L-Tryptophan

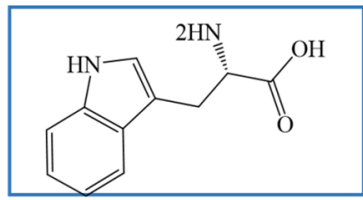

L-Phenylalanine

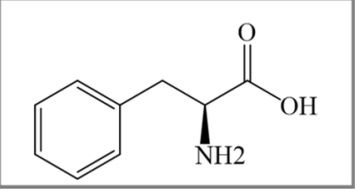

L-Tyrosine

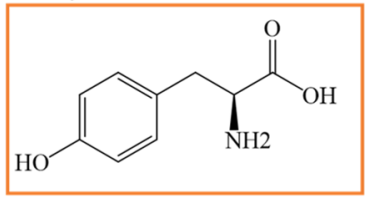

L-Dopa

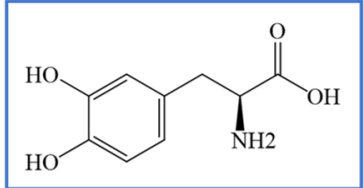

Figure 1. Chemical structures of the investigated ILs and amino acids.

times combined with $\left[\mathrm{BF}_{4}\right]^{-}$, which may raise some toxicity and biodegradability concerns. ${ }^{8}$ The ample flexibility of ILs should, however, permit the preparation of new compounds with an acceptable environmental footprint and biocompatibility to be used in the creation of novel ABSs. Cholinium-based ILs are a promising family of ILs that can be prepared from natural sources and may present high biodegradability and, in some cases, marginal (eco)toxicity. ${ }^{9-11}$ In particular, cholinium chloride is an essential nutrient with an important role, namely, as a precursor in the synthesis of vitamins (e.g., thiamine) and enzymes that participate in the carbohydrates' metabolism. ${ }^{12,13}$ Taking into account their advantageous features, several works have been published reporting the use of cholinium-based ILs to form ABSs with salts. Xie et al. ${ }^{14}$ investigated novel ABSs formed by four cholinium-based ILs comprising carboxylate anions and $\mathrm{K}_{3} \mathrm{PO}_{4}$ to extract tryptophan, phenylalanine, and caffeine. The authors reported novel ABS liquid-liquid phase diagrams and showed their enhanced extraction performance, as well as the presence of liquid-crystal structures in ABSs formed by ILs with long-alkyl-chain anions. Shahriari et al. ${ }^{15}$ determined the phase diagrams of novel ABSs formed by a series of cholinium-based ILs with different anions and $\mathrm{K}_{3} \mathrm{PO}_{4}$, having evaluated their performance to extract antibiotics. This type of ABS has also been used to extract antibiotics from real fermentation broths, ${ }^{16}$ and it was latter demonstrated that they represent a more economical alternative to recovering tetracycline from the fermentation broth than more traditional ABSs formed by polymers. $^{17}$

To design effective ABSs for separation processes, it is relevant to determine and characterize their phase diagrams. The respective ternary phase diagrams provide valuable data on the phase-forming components' compositions required to form two-phase systems and on the composition of each phase for a given overall mixture. Accordingly, a significant number of works have focused on the determination of novel IL-based ABS ternary phase diagrams. ${ }^{8}$ Furthermore, it is well-known that ILs comprising long alkyl side chains tend to self-aggregate in aqueous media, a trend that was further demonstrated to occur in $\mathrm{ABSs}^{18}$ and that may lead to unfavorable extraction results. ${ }^{19}$ In a previous work, the impact of the IL cation alkyl side chain length on the two-phase separation and creation of ABSs has been comprehensively investigated by employing the 1-alkyl-3methylimidazolium chloride series, with both odd and even alkyl side chains. ${ }^{20}$ An odd-even effect was identified on the Setschenow salting-out coefficient $\left(k_{\mathrm{s}}\right)$, which quantitatively addresses the two-phase formation capacity, as a function of the number of methylene groups at the longest IL cation alkyl side chain. ${ }^{20}$ ILs comprising cations with even alkyl side chains have higher molar volumes and are thus more easily salted out, meaning that they more easily undergo phase separation in aqueous media. Although this trend was detected for the IL cation, the effect of the IL anion on ABS formation is scarcely studied. Some reports exist on the effect of the IL anion alkyl chain length on the formation of ABSs, ${ }^{14,21-24}$ but most of these studies employed ILs with chemically different anions or only with even alkyl chains at the anion. In general, it is understood that ILs with longer aliphatic moieties at the anion are more easily salted out and need less inorganic salt to undergo phase separation. However, no studies on the self-aggregation impact of long-alkyl-chain anion ILs and on the possibility of an oddeven effect have been performed. Furthermore, to the best of our knowledge, the impact of the odd-even effect in the partitioning of target molecules in ABSs was never investigated. It should be remarked that, although the odd-even effect is scarcely studied in aqueous solutions, the IL odd-even effect was already reported in a series of neat IL properties, such as molar volumes, viscosities, and entropy and enthalpy of vaporization. ${ }^{25-28}$ Santos and co-workers, ${ }^{26-28}$ by studying several series of imidazolium-based ILs with cations of different alkyl-side-chain lengths, demonstrated that the odd-even effect is a result of the IL nanosegregation and consequent impact on these fluids' cohesive energy.

Aiming at a better understanding of the influence of the IL anion alkyl chain length on the creation of ABSs, in this work, seven cholinium-based ILs with carboxylate anions, from acetate to octanoate and comprising both even and odd alkyl side chains ( $[\mathrm{Ch}]\left[\mathrm{C}_{n} \mathrm{CO}_{2}\right]$, where $\left.n=1-7\right)$, were synthesized and used in ABS formation with dipotassium hydrogen phosphate $\left(\mathrm{K}_{2} \mathrm{HPO}_{4}\right)$. The corresponding ternary phase diagrams were determined at $298 \pm 1 \mathrm{~K}$, including tie lines (TLs) and critical points, and further evaluated in terms of their extraction capability for amino acids, namely, L-tryptophan, L-phenylalanine, L-tyrosine, and L-3,4-dihydroxyphenylalanine/L-dopa. The Setschenow coefficient of each ABS was determined, and the odd-even effect was addressed in both ABS formation and amino acid partitioning between the coexisting phases.

\section{MATERIALS AND METHODS}

2.1. Materials. $\mathrm{ABS}$ s were prepared using aqueous solutions of potassium phosphate $\left(\mathrm{K}_{2} \mathrm{HPO}_{4} ;>98 \mathrm{wt} \%\right.$ purity $)$, purchased from Sigma-Aldrich, and aqueous solutions of each IL. The investigated cholinium-based ILs comprise anions derived from carboxylic acids, corresponding to cholinium acetate ([Ch]$\left[\mathrm{C}_{1} \mathrm{CO}_{2}\right] ;>98$ wt $\%$ purity) from Iolitec and cholinium 
propanoate $\left([\mathrm{Ch}]\left[\mathrm{C}_{2} \mathrm{CO}_{2}\right]\right)$, cholinium butanoate $([\mathrm{Ch}]$ $\left.\left[\mathrm{C}_{3} \mathrm{CO}_{2}\right]\right)$, cholinium pentanoate $\left([\mathrm{Ch}]\left[\mathrm{C}_{4} \mathrm{CO}_{2}\right]\right)$, cholinium hexanoate $\left([\mathrm{Ch}]\left[\mathrm{C}_{5} \mathrm{CO}_{2}\right]\right)$, cholinium heptanoate $([\mathrm{Ch}]$ $\left.\left[\mathrm{C}_{6} \mathrm{CO}_{2}\right]\right)$, and cholinium octanoate $\left([\mathrm{Ch}]\left[\mathrm{C}_{7} \mathrm{CO}_{2}\right]\right)$ that were synthesized in our laboratory following protocols previously described. ${ }^{29}$ All ILs synthesized showed high purity (>97 wt \% purity; ${ }^{1} \mathrm{H}$ and ${ }^{13} \mathrm{C}$ NMR results are given in the Supporting Information, SI). The required precursors, namely, a cholinium hydroxide solution (46 wt \%, Sigma-Aldrich), propanoic, butanoic, pentanoic, hexanoic, and heptanoic acids (99 wt \% purity, Acros Organics), and octanoic acid (98 wt \% purity, Sigma-Aldrich), were commercially acquired. Briefly, the corresponding acids were added dropwise to an aqueous solution of $[\mathrm{Ch}][\mathrm{OH}](1.1$ equiv of acid) at $298 \mathrm{~K}$, with the resultant mixture being stirred at room temperature and under an inert atmosphere for at least $12 \mathrm{~h}$ to produce the choliniumbased ILs. The mixtures were then dried for $4 \mathrm{~h}$ under vacuum. The remaining acid was removed by extraction with ethyl acetate. Residual solvents were removed under vacuum, and the prepared ILs were finally dried under high vacuum (ca. $72 \mathrm{~h}$ ).

The water used was double-distilled, passed by a reverse osmosis system, and treated with a Milli-Q Plus 185 water purification apparatus. For partition studies, four amino acids were investigated, namely, L-phenylalanine (99.0 wt \% purity) purchased from Alfa Aesar, L-tyrosine (99.0 wt \% purity) purchased from Fluka, and L-tryptophan ( $99.0 \mathrm{wt} \%$ purity) and L-3,4-dihydroxyphenylalanine or L-dopa (98.0 wt \% purity) acquired from Sigma-Aldrich. The chemical structures of the investigated amino acids are given in Figure 1.

2.2. Phase Diagrams, Critical Points, and Setschenow Coefficients. Binodal data were determined by cloud-point titration at $298 \pm 1 \mathrm{~K}$ and atmospheric pressure, following a previously described method. ${ }^{21}$ Aqueous solutions of the inorganic salt at $40 \mathrm{wt} \%$ and aqueous solutions of the different ILs from 60 to $80 \mathrm{wt} \%$ were used. Each point of the binodal curve was determined by weight $\left( \pm 10^{-4} \mathrm{~g}\right)$. The experimental solubility curves were fitted by the equation originally described by Merchuk et al.: ${ }^{30}$

$$
[\mathrm{IL}]=A \exp \left\{\left(B[\text { Salt }]^{0.5}\right)-\left(C[\text { Salt }]^{3}\right)\right\}
$$

where $A, B$, and $C$ are constants obtained by the regression, and [IL] and [Salt] correspond to the IL and salt weight fraction percentages, respectively.

TLs associated with each binodal curve, i.e., the composition of each phase for a target mixture composition, were determined by a gravimetric method. ${ }^{30}$ Several ternary mixtures constituted by $\mathrm{IL}+\mathrm{K}_{2} \mathrm{HPO}_{4}+$ water at the biphasic region were prepared by weight and vigorously mixed. Each mixture was centrifuged for $30 \mathrm{~min}$ at $298 \pm 1 \mathrm{~K}$, aiming for the separation of the two phases. Both phases were carefully separated and weighed. Each TL was determined by the lever-arm rule ${ }^{30}$ according to eqs 2-5:

$$
\begin{aligned}
& {[\mathrm{IL}]_{\mathrm{T}}=A \exp \left\{\left(B[\text { Salt }]_{\mathrm{T}}^{0.5}\right)-\left(C[\text { Salt }]_{\mathrm{T}}{ }^{3}\right)\right\}} \\
& {[\mathrm{IL}]_{\mathrm{B}}=A \exp \left\{\left(B[\text { Salt }]_{\mathrm{B}}^{0.5}\right)-\left(C[\text { Salt }]_{\mathrm{B}}{ }^{3}\right)\right\}} \\
& {[\mathrm{IL}]_{\mathrm{T}}=\frac{[\mathrm{IL}]_{\mathrm{M}}}{\alpha}-\frac{1-\alpha}{\alpha}[\mathrm{IL}]_{\mathrm{B}}} \\
& {[\text { Salt }]_{\mathrm{T}}=\frac{[\text { Salt }]_{\mathrm{M}}}{\alpha}-\frac{1-\alpha}{\alpha}[\text { Salt }]_{\mathrm{B}}}
\end{aligned}
$$

where the subscripts $M, T$, and B represent the initial mixture and the top and bottom phases, respectively. $\alpha$ corresponds to the ratio between the weight of the top phase and the total mass of the system. In all systems, the top phase is enriched in IL (ILrich phase), whereas the salt is majorly enriched in the bottom phase.

Each TL length (TLL) was determined by the following equation:

$$
\mathrm{TLL}=\sqrt{\left([\text { Salt }]_{\mathrm{T}}-[\text { Salt }]_{\mathrm{B}}\right)^{2}+\left([\mathrm{IL}]_{\mathrm{T}}-[\mathrm{IL}]_{\mathrm{B}}\right)^{2}}
$$

The critical point of each system, i.e., when the compositions of the two aqueous phases are identical, was determined through application of the Sherwood method using the TL slopes, ${ }^{31}$ according to eq 7

$$
[\mathrm{IL}]=f[\text { Salt }]+g
$$

where $f$ and $g$ were obtained from the fitting and [IL] and [Salt] are the weight fraction percentages of the IL and salt.

The magnitude of salting out may be quantified by correlating the solubility values with the empirical equation of Setschenow. ${ }^{32}$ In this work, the modified version of the original Setschenow equation proposed by Hey et al., ${ }^{33}$ previously used to characterize polymer-based $\mathrm{ABSs}^{34}$ and IL-salt-based ABSs, ${ }^{34,35}$ was applied:

$$
\ln \frac{[\mathrm{IL}]_{\mathrm{T}}}{[\mathrm{IL}]_{\mathrm{B}}}=k_{\mathrm{IL}}\left([\mathrm{IL}]_{\mathrm{B}}-[\mathrm{IL}]_{\mathrm{T}}\right)+k_{\mathrm{s}}\left([\mathrm{Salt}]_{\mathrm{B}}-[\mathrm{Salt}]_{\mathrm{T}}\right)
$$

where $\mathrm{T}$ and $\mathrm{B}$ designate the top (IL-rich) and bottom (saltrich) phases, respectively, and [IL] and [Salt] correspond to the molality of the IL and salt. The $k_{\mathrm{IL}}$ and $k_{\mathrm{s}}$ parameters are related with the IL activity coefficient to its concentration and the salting-out coefficient, respectively.

2.3. Cation-Anion Interaction Energies. The cationanion total interaction energies of each IL, $E_{\text {Int }}$ were determined using the COSMO-RS thermodynamic model. This model combines quantum chemistry, based on the dielectric continuum model known as COSMO (COnductor-like Screening MOdel for Real Solvents), with statistical thermodynamic calculations. The process employed in this work to determine the interaction energies was described by Kurnia et al. ${ }^{36}$ The COSMO quantum-chemical calculations were performed with the TURBOMOLE 6.1 program package at the density functional theory level. The BP functional B88-P86 with a triple-z valence-polarized basis set (TZVP) and the resolution of identity (RI) standard approximation were applied. ${ }^{37}$ The COSMOtherm $X$ program and the parameter file BP_TZVP_C20_0111 (COSMOlogic GmbH \& Co. KG, Leverkusen, Germany) were used. ${ }^{38}$

2.4. Extraction of Amino Acids. ABSs containing amino acid aqueous solutions $\left(5 \mathrm{~g} \cdot \mathrm{L}^{-1}\right.$ for L-tryptophan, $15 \mathrm{~g} \cdot \mathrm{L}^{-1}$ for $\mathrm{L}-$ phenylalanine, $2.5 \mathrm{~g} \cdot \mathrm{L}^{-1}$ for L-tyrosine, and $1.5 \mathrm{~g} \cdot \mathrm{L}^{-1}$ for L-dopa, defined according to the amino acid solubility in water) were prepared and used to study the amino acid partitioning between the ABS coexisting phases. The compositions of the ternary systems applied in the partitioning experiments were defined according to the determined phase diagrams and TLs. To circumvent inconsistencies in the results that could result from the different phase compositions and with the goal of addressing the odd-even effect in the amino acids partitioning, all extraction studies were carried out at a constant TLL (ca. 58 \pm 2 ). Each mixture was vigorously stirred to allow equilibration and then centrifuged for $30 \mathrm{~min}$ at $298 \mathrm{~K}$ to reach complete separation between the coexisting phases. Both phases were 

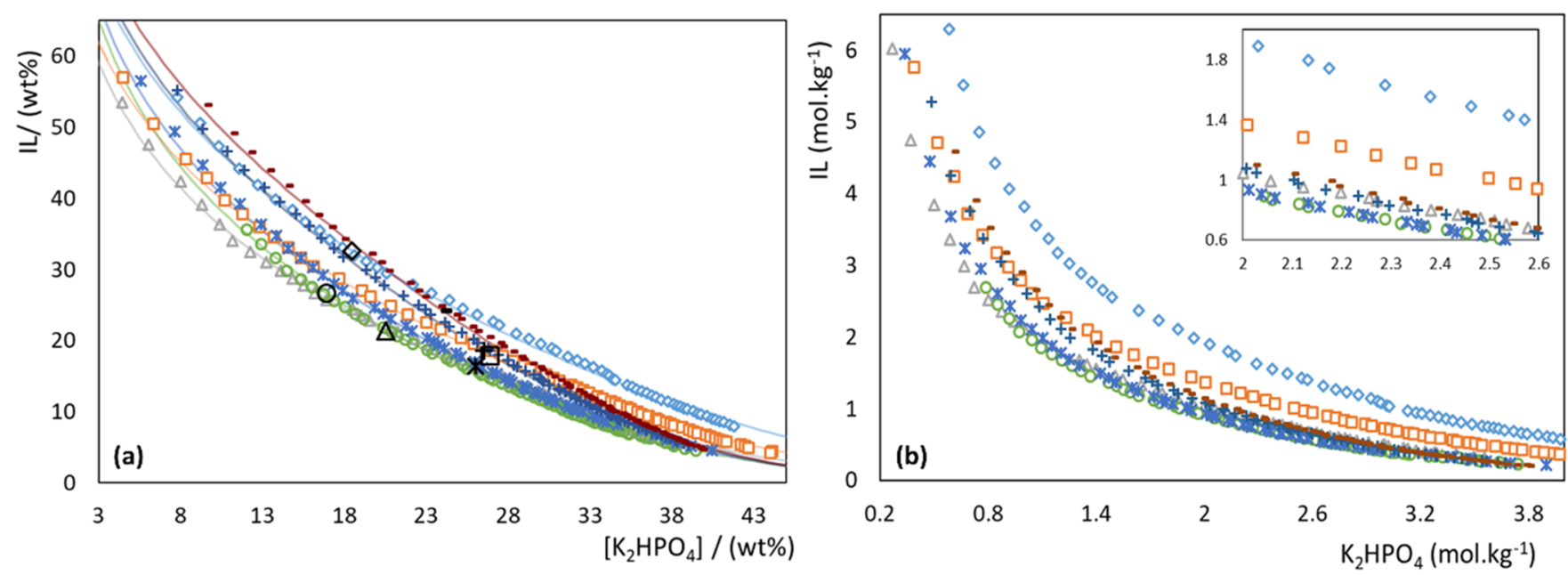

Figure 2. Phase diagrams in an orthogonal representation in (a) weight fraction and (b) molality units for the ABSs formed by cholinium-based ILs + $\mathrm{K}_{2} \mathrm{HPO}_{4}+$ water at $298 \pm 1 \mathrm{~K}$ and atmospheric pressure. ILs: $[\mathrm{Ch}]\left[\mathrm{C}_{1} \mathrm{CO}_{2}\right]$ (blue $\left.\diamond\right),[\mathrm{Ch}]\left[\mathrm{C}_{2} \mathrm{CO}_{2}\right]$ (orange $\square$ ), $[\mathrm{Ch}]\left[\mathrm{C}_{3} \mathrm{CO}{ }_{2}\right](\mathrm{gray} \triangle \mathrm{C}$ ), $[\mathrm{Ch}]\left[\mathrm{C}_{4} \mathrm{CO}_{2}\right]$ (green $\mathrm{O}$ ), $[\mathrm{Ch}]\left[\mathrm{C}_{5} \mathrm{CO}_{2}\right]$ (blue *), $[\mathrm{Ch}]\left[\mathrm{C}_{6} \mathrm{CO}_{2}\right]$ (blue + ), $[\mathrm{Ch}]\left[\mathrm{C}_{7} \mathrm{CO}_{2}\right]$ (red bar). The lines correspond to the fitting by eq 1 , and larger symbols correspond to the critical point of each $\mathrm{ABS}$.

separated, and the amount of amino acid in each phase was quantified by UV spectroscopy, using a BioTeck Synergy HT microplate reader with the calibration curves previously determined (at a wavelength of $280 \mathrm{~nm}$ for L-tryptophan, Ltyrosine, and L-dopa and at $260 \mathrm{~nm}$ for L-phenylalanine). At least three ABSs for each amino acid were prepared, and at least three samples of each phase were quantified.

The ABS's capability to extract different amino acids to the ILrich phase was evaluated by its partition coefficient $\left(K_{\mathrm{AA}}\right)$. This parameter corresponds to the ratio of the concentration of each amino acid in the phase enriched in IL to that in the salt-rich phase, according to eq 9.

$$
K_{\mathrm{AA}}=\frac{[\mathrm{AA}]_{\mathrm{IL}}}{[\mathrm{AA}]_{\text {Salt }}}
$$

where $[\mathrm{AA}]_{\mathrm{IL}}$ and $[\mathrm{AA}]_{\mathrm{Salt}}$ correspond to the concentration of each amino acid (AA) in the phases enriched in IL and salt, respectively.

The $\mathrm{pH}$ of the IL- and $\mathrm{K}_{2} \mathrm{HPO}_{4}$-rich aqueous phases was determined at $298 \mathrm{~K}$ using Mettler Toledo S47 SevenMulti dual meter $\mathrm{pH}$ /conductivity equipment (data are shown in the SI).

\section{RESULTS AND DISCUSSION}

3.1. Phase Diagrams and Critical Points. New ternary phase diagrams of ABSs composed of water, $\mathrm{K}_{2} \mathrm{HPO}_{4}$, and cholinium-based ILs with carboxylate anions of different alkyl chain lengths were determined in this work at $298 \pm 1 \mathrm{~K}$. Their orthogonal representations, both in percentage of weight fraction and in molality units, are shown in Figure 2. The weight fraction detailed results are given in Tables S1-S7. Experimental binodal data were fitted by eq 1, whose fitting is given in Figure 2. The fitted constants $A, B$, and $C$ were estimated by the least-squares regression, with their values being given in Table S8. At least four TLs and their respective lengths (TLLs) were determined for each system by eqs $2-6$, with detailed results shown in Table S9. In addition, the critical point of each system was determined, being provided in Figure 2 (cf. Table S10 for detailed data).

Because all ILs are composed of a common cholinium cation, the results depicted in Figure 2 mirror the effect of the IL anion on the formation of an $\mathrm{ABS}$ with $\mathrm{K}_{2} \mathrm{HPO}_{4}$. The two-phase region is located above the solubility curve, and the larger this region is, the more prone the $\mathrm{IL}$ is to being salted out by $\mathrm{K}_{2} \mathrm{HPO}_{4}$ and to form an ABS. It is well documented ${ }^{39}$ that small IL anions derived from organic acids are excellent hydrogen-bond acceptors and exhibit a high capability of establishing hydrogen bonds with water. Furthermore, the hydroxyl group at the cholinium cation can establish strong hydrogen bonds or dipole-dipole interactions with water molecules. ${ }^{40,41}$ Therefore, cholinium carboxylate ILs display a high affinity for water and only moderate-to-strong salting-out salts are able to create ABS. Accordingly, it was previously shown that these ILs form an $\mathrm{ABS}$ with $\mathrm{K}_{3} \mathrm{PO}_{4}{ }^{14}$ and in this work, we show that they form an $\mathrm{ABS}$ with $\mathrm{K}_{2} \mathrm{HPO}_{4}$. However, the studied cholinium carboxylate ILs do not form ABSs with weaker salting-out salts, such as $\mathrm{KH}_{2} \mathrm{PO}_{4}$ or $\mathrm{K}_{2} \mathrm{HPO}_{4} / \mathrm{KH}_{2} \mathrm{PO}_{4}$ at $\mathrm{pH} 7$ (as experimentally attempted by us in this work; data not shown).

The investigated ILs' capacity to form an ABS at 22 wt \% $\mathrm{K}_{2} \mathrm{HPO}_{4}$ follows the order: $[\mathrm{Ch}]\left[\mathrm{C}_{1} \mathrm{CO}_{2}\right] \approx[\mathrm{Ch}]\left[\mathrm{C}_{7} \mathrm{CO}_{2}\right]<$ $[\mathrm{Ch}]\left[\mathrm{C}_{6} \mathrm{CO}_{2}\right]<[\mathrm{Ch}]\left[\mathrm{C}_{2} \mathrm{CO}_{2}\right]<[\mathrm{Ch}]\left[\mathrm{C}_{5} \mathrm{CO}_{2}\right]<[\mathrm{Ch}]-$ $\left[\mathrm{C}_{3} \mathrm{CO}_{2}\right] \approx[\mathrm{Ch}]\left[\mathrm{C}_{4} \mathrm{CO}_{2}\right]$. When weight fraction is used (Figure 2a), a straight relationship of the ILs' aptitude to be salted out cannot be straightforwardly assessed. When using the molality units, the differences arising from the different molecular weights of the ILs are circumvented, consenting a more accurate analysis of the effect of the several phase-forming components on endorsing liquid-liquid demixing. The binodal curves represented in the molality units, shown in Figure $2 b$, prove that the ILs' performance to create ABSs, e.g., at $2.0 \mathrm{~mol}$. $\mathrm{kg}^{-1}$ of $\mathrm{K}_{2} \mathrm{HPO}_{4}$, follows the rank $[\mathrm{Ch}]\left[\mathrm{C}_{1} \mathrm{CO}_{2}\right]<[\mathrm{Ch}]$ $\left[\mathrm{C}_{2} \mathrm{CO}_{2}\right]<[\mathrm{Ch}]\left[\mathrm{C}_{7} \mathrm{CO}_{2}\right] \approx[\mathrm{Ch}]\left[\mathrm{C}_{6} \mathrm{CO}_{2}\right]<[\mathrm{Ch}]\left[\mathrm{C}_{3} \mathrm{CO}_{2}\right]<$ $[\mathrm{Ch}]\left[\mathrm{C}_{4} \mathrm{CO}_{2}\right] \approx[\mathrm{Ch}]\left[\mathrm{C}_{5} \mathrm{CO}_{2}\right]$. This order shows that the capability of ABS formation (or of the IL to be salted out) increases with an increase in the size of the aliphatic moiety at the IL anion, at least up to $[\mathrm{Ch}]\left[\mathrm{C}_{5} \mathrm{CO}_{2}\right]$, in which the ILs' selfaggregation is not yet playing a role. When self-aggregation of the ILs occurs, there is a decrease in their ability to create ABSs, as observed for $[\mathrm{Ch}]\left[\mathrm{C}_{6} \mathrm{CO}_{2}\right]$ (cholinium heptanoate) and $[\mathrm{Ch}]\left[\mathrm{C}_{7} \mathrm{CO}_{2}\right]$ (cholinium octanoate). Both ILs have alkyl chains sufficiently long to self-aggregate in aqueous media at 298 $\mathrm{K}$ [critical micellar concentration (CMC) values: $410 \pm 13 \mathrm{mM}$ 


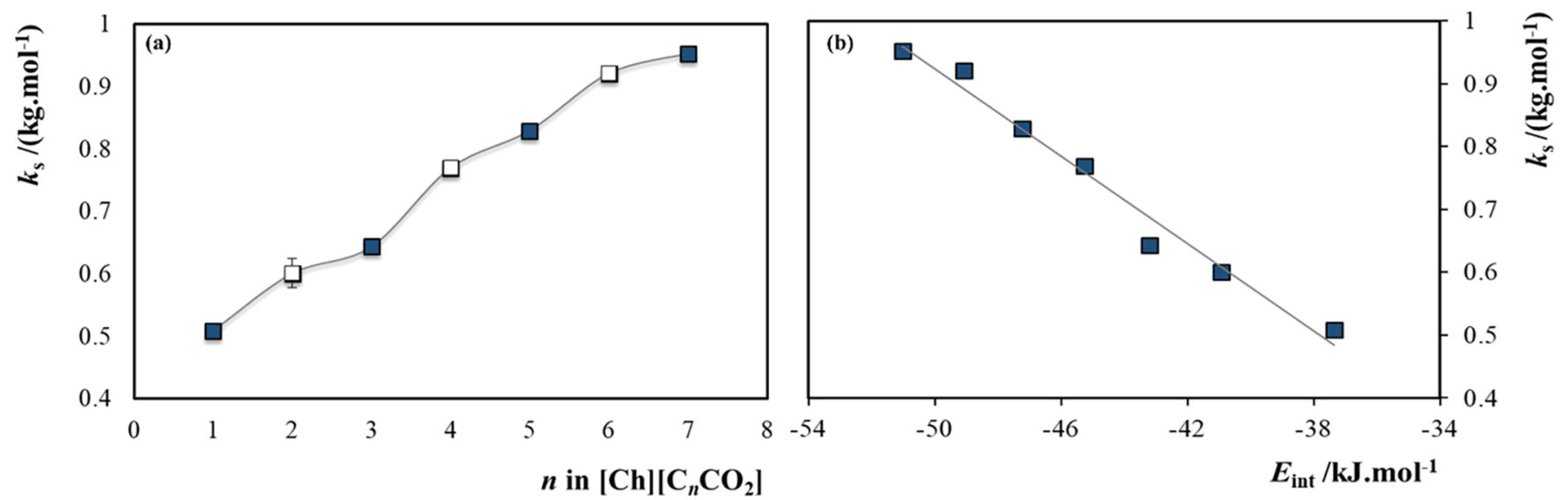

Figure 3. Salting-out coefficients $\left(k_{\mathrm{s}}\right)$ as a function of the IL anion alkyl chain length, $n$, in the $[\mathrm{Ch}]\left[\mathrm{C}_{n} \mathrm{CO}_{2}\right]$ ILs, and of the cation-anion total interaction energies of each IL $\left(E_{\text {int }}\right)$.

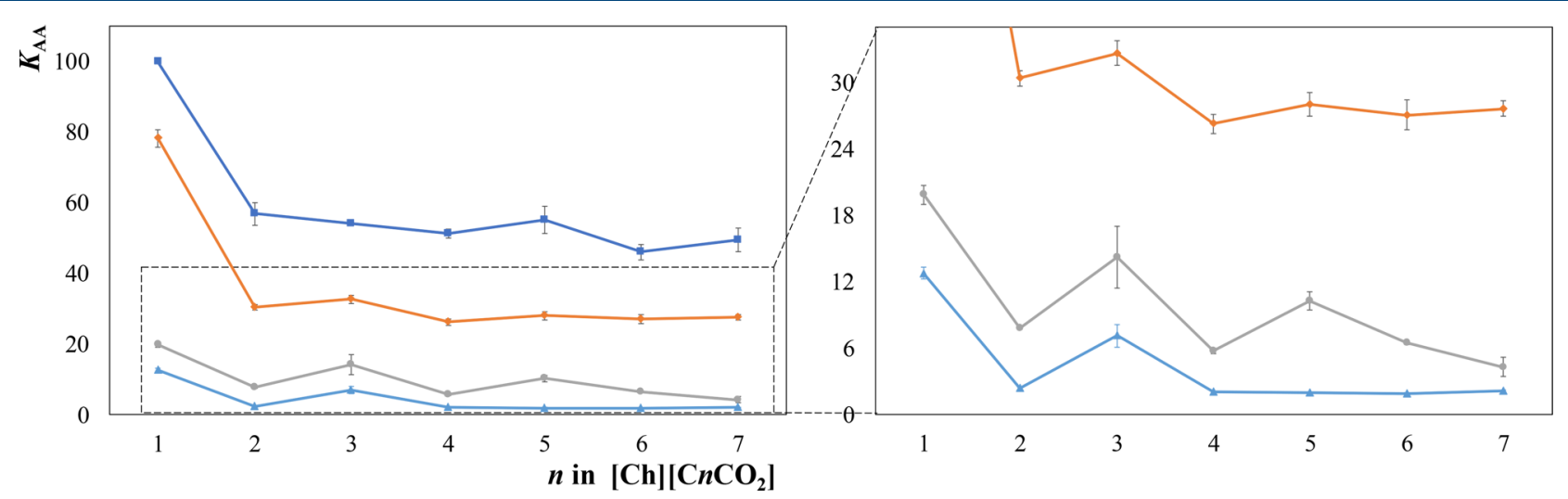

Figure 4. Partition coefficients $\left(K_{\mathrm{AA}}\right)$ of the studied ABSs at $298 \mathrm{~K}$ for L-tryptophan (blue $\boldsymbol{\square}$ ), L-phenylalanine (orange $\bullet$ ), L-tyrosine (gray O), and Ldopa (blue $\mathbf{\Lambda})$.

for $[\mathrm{Ch}]\left[\mathrm{C}_{6} \mathrm{CO}_{2}\right]$ and $383.0 \mathrm{mM}$ for $\left.[\mathrm{Ch}]\left[\mathrm{C}_{7} \mathrm{CO}_{2}\right]^{42}\right]$. Details on the CMC determination are given in the SI. This trend in the $\mathrm{ABS}$ formation is in agreement with what has been previously observed for the IL cation effect ${ }^{20}$ and seems to be independent of the salt used.

The phase separation in ABS is a result of the salting out of the salt over the IL in aqueous media, which can be quantified by the Setschenow salting-out coefficient, $k_{s}$. The Setschenow constant of each IL-based ABS was determined by the simultaneous regression of at least four TLs, at initial mixture compositions within the two-phase region. The corresponding values are provided in Table S11. Figure 3 depicts the behavior of the salting-out coefficient, $k_{\mathrm{s}}$, along the IL anion alkyl chain length. Although there is a continuous increase of the salting-out coefficient with the size of the IL anion aliphatic moiety, as expected because of the IL anion hydrophobicity increase, a subtle odd-even effect is identified in these results. A slightly higher value of the salting-out coefficient is visible for ILs comprising anions with even alkyl chains, meaning that these are more prone to be salted out by $\mathrm{K}_{2} \mathrm{HPO}_{4}$ or more easily undergo phase separation in aqueous solutions. The current results are in good agreement with our previous findings on the effect of the IL cation alkyl chain in ABS formation using the $\left[\mathrm{C}_{n} \mathrm{C}_{1} \mathrm{im}\right] \mathrm{Cl}(n=$ $2-12$ ) IL series and $\mathrm{K}_{2} \mathrm{CO}_{3} .{ }^{20}$ In this previous work, ${ }^{20}$ the oddeven effect in the $k_{\mathrm{s}}$ values was, however, more significant, and particularly in ILs up to $n=6$, where the nanostructuration/ nanosegregation has a less relevant impact. This difference in the intensity is thus connected to the cation versus anion effect toward the IL nanostructuration. The odd-even effect has been previously shown in some IL properties, e.g. viscosities, molar volumes, and entropy and enthalpy of vaporization. ${ }^{25-28}$ It is demonstrated here that it also occurs in aqueous solution, indicating that the ILs' organization and their nanostructuration in aqueous media are responsible for the observed trend. When dealing with ILs' pure properties, the odd-even effect was elucidated as a result of the orientation of the terminal methyl groups and further influence on the ILs' cohesive energy, ${ }^{26-28}$ which is here shown to have a direct effect on their behavior in aqueous media and is visible also as a result of the IL anion aliphatic moiety.

In opposition to that observed with the IL cation impact on the ABS formation aptitude, which is mostly governed by steric and entropic contributions, ${ }^{21}$ the IL anion effect on the formation of two-phase systems is primarily governed by favorable (or nonfavorable) interactions with water. Anions are classically more polarizable than cations because of their more diffuse valence electronic configuration; thus, their hydration is more significant than that of the cations. ${ }^{43}$ The anions' propensity to form hydration complexes is directly connected to their cation-anion interaction strength. Accordingly, there is a close correlation between the salting-out coefficient and the total cation-anion energy of each IL, which in this work was determined by COSMO-RS, as shown in Figure 3. The odd-even effect observed is a direct result of the $k_{s}$ oddeven effect itself. It should be remarked that the cation-anion total interaction energies of each IL are good indicators of the 
complexity of the interactions taking place in ABSs, particularly van der Waals, electrostatic, and hydrogen-bonding interactions, because the same types of interactions occur in the neat ILs, contributing to their cohesive energy.

3.2. Amino Acid Partitioning Behavior. The potential of ABS for extracting target compounds depends on the possibility of manipulating the properties of the coexisting phases, which can be achieved by changing the IL used in the ABS formulation. In this work, the potential of ABSs formed by cholinium carboxylate ILs and $\mathrm{K}_{2} \mathrm{HPO}_{4}$ to act as separation platforms for hydrophobic amino acids, namely, L-tryptophan, L-phenylalanine, L-tyrosine, and L-dopa, was evaluated by their partition coefficients. These aromatic amino acids, and thus hydrophobic amino acids, were chosen to identify differences in partitioning that could mainly arise from the dispersive interactions established with the IL anion aliphatic moieties. The results obtained are given in Figure 4. The extraction efficiencies of these systems are provided in the SI, as well as the respective results for both parameters. All partition studies were carried out at a common TLL (ca. $58 \pm 2)$ to prevent the effects that could derive from the TLL effect or differences in the composition between the two phases.

ABSs constituted of cholinium carboxylate ILs and $\mathrm{K}_{2} \mathrm{HPO}_{4}$ present a satisfactory extraction ability for amino acids. In all investigated ABSs, amino acids favorably partition to the most hydrophobic phase - the phase enriched in IL-with extraction efficiencies ranging from $66.8 \%$ to $99.9 \%$ (cf. the SI). The amino acid partition coefficients $\left(K_{\mathrm{AA}}\right)$, depicted in Figure 4, range between 1.88 and complete extraction. In Figure 4, a $K_{\mathrm{AA}}$ value of 100 is shown for the cases where complete extraction was achieved. These high values in both the partition coefficients and extraction efficiencies are due to the salting-out effect exerted by $\mathrm{K}_{2} \mathrm{HPO}_{4}$ and favorable interactions established between the ILrich phase-forming components and amino acids. ZafaraniMoattar and Hamzehzadeh ${ }^{44}$ investigated the separation of several amino acids (L-tryptophan, L-phenylalanine, L-tyrosine, L-leucine, and L-valine) using $\mathrm{ABS}$ formed by $\left[\mathrm{C}_{4} \mathrm{mim}\right] \mathrm{Br}$ and potassium citrate. Authors ${ }^{44}$ concluded that dispersive interactions are the driving force for the amino acid partition. The results obtained for a given IL or ABS agree with the findings of Zafarani-Moattar and Hamzehzadeh ${ }^{44}$ because the extraction efficiencies (Table S12 and Figure A3) and partition coefficients (Table S13 and Figure 4) follow the octanol-water partition coefficients of each amino acid $\left[\log \left(K_{\mathrm{ow}}\right)\right.$ : L-tryptophan = -1.06 ; L-phenylalanine $=-1.38$; L-tyrosine $=-2.26$; $\mathrm{L}$-dopa $=$ $-2.74] .{ }^{45}$ In general, more hydrophobic amino acids are better extracted to the most hydrophobic phase, i.e., the phase enriched in IL. However, ABSs composed of ILs with shorter aliphatic moieties have a better performance at extracting amino acids to the IL-rich phase, meaning that hydrophobic interactions are not the only factor ruling the partition of a given amino acid along the $[\mathrm{Ch}]\left[\mathrm{C}_{n} \mathrm{CO}_{2}\right]$ series of ILs. This trend shows that the partitioning results are associated with each amino acid affinity for each phase, which is ruled by multiple effects, including salting-out effects and hydrogen-bonding, dispersive, and electrostatic interactions.

In addition to the two-phase formation capacity, the occurrence of an even-odd effect is also observed in the partition coefficient results along the size of the anion aliphatic moiety (Figure 4). The odd-even effect occurs in the partition coefficients of all amino acids. Overall, ABSs constituted of ILs with odd alkyl chains at the anion lead to slightly higher partition coefficients. The obtained results suggest that the salting-out effect exerted by the salt is not the main factor ruling the amino acid partition because higher $k_{\mathrm{s}}$ values are observed for ABSs composed of ILs with even alkyl chains at the anion (Figure 3). Taking into account the previous discussion, there is thus a multitude of simultaneous effects governing the amino acid partitioning to the IL-rich phase, where salting-out effects and noncovalent interactions occurring between the amino acids and the ABS phase-forming components have a significant impact. As observed in several properties of pure ILs, ${ }^{26-28}$ the orientation of the terminal methyl groups of the carboxylate anion and their impact on the cohesive energy directly influence the ILs' ability to interact with amino acids, which may thus be responsible for the observed odd-even effect.

Besides the previously reported odd-even effect in the thermophysical and thermodynamic properties of neat ILs, ${ }^{26-28}$ a similar phenomenon is shown here to occur in aqueous solutions of ILs, namely, in ABSs constituted of ILs and salts, and that it is visible also as a result of the IL anion. The gathered data allow confirmation of the existence of an odd-even effect derived from the IL anion alkyl chain length, with an impact on both the two-phase or ABS formation ability and partitioning behavior of biomolecules.

\section{CONCLUSIONS}

ABSs comprising ILs have been largely studied as separation routes, demonstrating their outstanding performance on the extraction and purification of a diversity of biomolecules. However, to achieve optimal extraction and separation performance, a priori characterization of the respective ABS ternary phase diagrams is required. In this work, we determined the ABS phase diagrams, formed by water, cholinium carboxylate ILs, and $\mathrm{K}_{2} \mathrm{HPO}_{4}$, and evaluated their capacity to extract amino acids. The $[\mathrm{Ch}]\left[\mathrm{C}_{n} \mathrm{CO}_{2}\right]$ IL series, with $n=1-7$, was chosen to appraise the existence of odd-even effects. It is demonstrated here that this phenomenon occurs in both the ABS formation ability and extraction performance. ILs comprising even alkyl chains display slightly higher $k_{\mathrm{s}}$ values, meaning that these ILs are more prone to being salted out or more easily phase separated. The phase-separation ability in ABSs comprising ILs and salts is largely controlled by the IL anion capability to form hydration complexes, largely depending on the interactions established between the IL anion and water, as demonstrated by the correlation found between $k_{\mathrm{s}}$ and the total cation-anion IL interaction energy. Conversely, ABSs formed by ILs with odd alkyl chains at the anion lead to slightly higher partition coefficients, demonstrating that the salting-out effect does not play a major role in defining the partitioning trend. In addition to the demonstrated and reported odd-even effects on several properties of pure ILs, the results obtained in this work confirm an odd-even effect induced by the IL anion alkyl chain length in aqueous media, valuable to a better appreciation of the molecular-level mechanisms behind the ABS formation ability and their extraction performance.

\section{ASSOCIATED CONTENT}

\section{S Supporting Information}

The Supporting Information is available free of charge on the ACS Publications website at DOI: 10.1021/acs.iecr.9b00663.

NMR spectra and data of cholinium-based ILs, weight fraction data for the binodal curves of the ABS formed by each $\mathrm{IL}, \mathrm{K}_{2} \mathrm{HPO}_{4}$, and water, adjusted parameters and respective standard deviations, weight fraction composi- 
tions, phase diagrams comprising the respective TL data required for determination of the critical point of each ABS, critical point of each ABS, salting-out coefficients, conductivity as a function of the IL concentration, extraction efficiencies, and partition coefficients (PDF)

\section{AUTHOR INFORMATION}

\section{Corresponding Author}

*Tel: +351-234-370200. Fax: +351-234-370084. E-mail: maragfreire@ua.pt.

\section{ORCID 우}

Sónia P. M. Ventura: 0000-0001-9049-4267

Iola F. Duarte: 0000-0003-4289-9256

Mara G. Freire: 0000-0001-8895-0614

\section{Notes}

The authors declare no competing financial interest.

\section{Biographies}

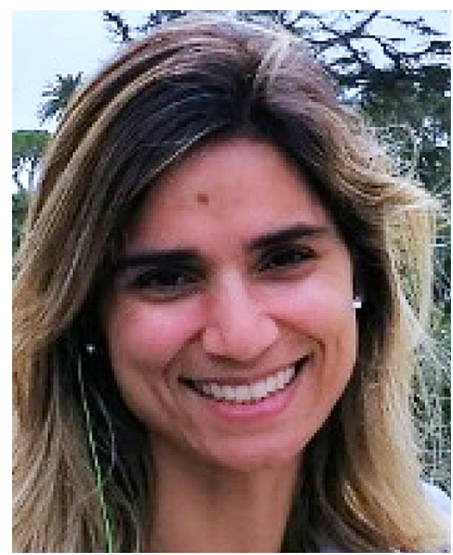

Diana Belchior graduated in 2014 in Bioprocess Engineering and Biotechnology from Federal University of Tocantins, Brazil, and received her M.Sc. degree in Biotechnology in 2016. Currently, she is a Ph.D. student in Chemical Engineering at the University of Aveiro, Portugal, working on the development of new platforms for the extraction and separation of biomolecules using ionic liquids.

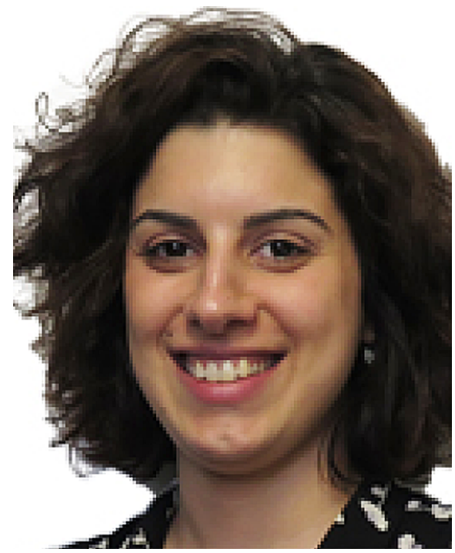

Mafalda Almeida obtained her Ph.D. in Biochemistry at University of Aveiro, Portugal. Since 2019, she has been a researcher at CICECOAveiro Institute of Materials, University of Aveiro, Portugal. Her main areas of research include the development and improvement of liquidliquid and/or solid-liquid techniques for the purification of biopharmaceuticals, such as antibodies, enzymes, and other recombinant proteins.

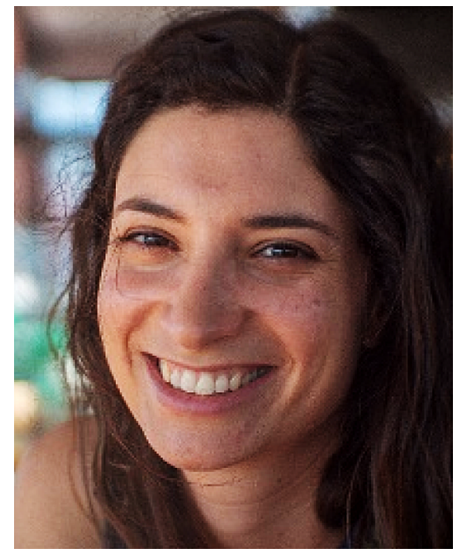

Tânia E. Sintra received her Ph.D. degree in 2017 in Chemistry from the University of Aveiro, Portugal. Since then, she has been a postdoctoral researcher at CICECO-Aveiro Institute of Materials, University of Aveiro, Portugal. Her main areas of research address the synthesis and characterization of sustainable ionic liquids for specific applications.

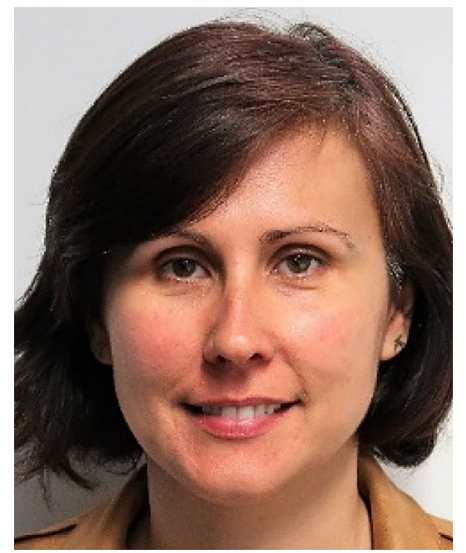

Sónia P. M. Ventura has a Ph.D. in Chemical Engineering and is an Assistant Professor at the Chemistry Department of University of Aveiro, Portugal, and member of CICECO-Aveiro Institute of Materials, University of Aveiro, Portugal. She has been working on the use of ecosolvents, namely, ionic liquids and deep eutectic solvents, in the design of tunable downstream processes toward the development of Marine Biorefinery supported by a Circular Economy strategy.

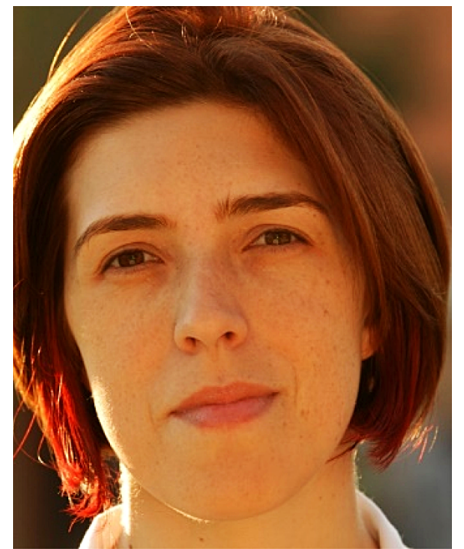

Iola F. Duarte has a Ph.D. in Chemistry and is a Principal Researcher at CICECO-Aveiro Institute of Materials, Department of Chemistry, University of Aveiro, Portugal. Her major domain of specialization is in the use of advanced analytical techniques, mainly NMR spectroscopy, for the comprehensive metabolic profiling of complex mixtures and biological samples. 


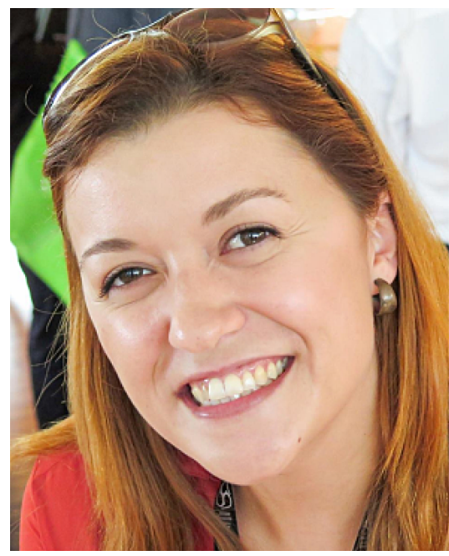

Mara G. Freire is a Coordinator Researcher at CICECO-Aveiro Institute of Materials, Chemistry Department, University of Aveiro, Portugal, the Coordinator of Group 5-Biomedic and Biomimetic Materials-within CICECO, and a member of the Young Scientists Seminar of the Lisbon Academy of Sciences. Her main research interests comprise the development of sustainable separation platforms for value-added compounds with therapeutic properties.

\section{ACKNOWLEDGMENTS}

This work was developed within the scope of the CICECOAveiro Institute of Materials, FCT, Project UID/CTM/50011/ 2019 and financed by national funds through the FCT/MCTES and Project POCI-01-0145-FEDER-030840 funded by FEDER, through COMPETE2020-Programa Operacional Competitividade e Internacionalização (POCI), and through FCT/ MCTES. D.C.V.B. and I.F.D. acknowledge Conselho Nacional de Desenvolvimento Científico e Tecnológico- $\mathrm{CNPq}$ for a Ph.D. grant (202337/2015-4) and FCT/MCTES for a research contract within the "Investigador FCT" Program. M.G.F. acknowledges the European Research Council under the European Union's Seventh Framework Programme (FP7/ 2007-2013)/ERC Grant Agreement 337753.

\section{REFERENCES}

(1) Marrucho, I. M.; Freire, M. G. Aqueous Biphasic Systems Based on Ionic Liquids for Extraction, Concentration and Purification Approaches. Ionic Liquids for Better Separation Processes. Green Chemistry and Sustainable Technology; Springer: Berlin, 2016.

(2) Albertsson, P. A. Partition of Cell Particles and Macromolecules; Wiley-Interscience: New York, 1986.

(3) Zaslavsky, B. Y. Aqueous Two-Phase Partitioning: Physical Chemistry and Bioanalytical Applications; Marcel Dekker: New York, 1995.

(4) Chen, J.; Spear, S. K.; Huddleston, J. G.; Holbrey, J. D.; Swatloski, R. P.; Rogers, R. D. Application of Poly(ethylene glycol)-based Aqueous Biphasic Systems as Reaction and Reactive Extraction Media. Ind. Eng. Chem. Res. 2004, 43, 5358.

(5) Asenjo, J. A.; Andrews, B. A. Aqueous Two-Phase Systems for Protein Separation: A Perspective. J. Chromatogr. A 2011, 1218, 8826. (6) Gutowski, K. E.; Broker, G. A.; Willauer, H. D.; Huddleston, J. G.; Swatloski, R. P.; Holbrey, J. D.; Rogers, R. D. Controlling the Aqueous Miscibility of Ionic Liquids: Aqueous Biphasic Systems of WaterMiscible Ionic Liquids and Water-Structuring Salts for Recycle, Metathesis, and Separations. J. Am. Chem. Soc. 2003, 125, 6632.

(7) Garcia-Chavez, L. Y.; Garsia, C. M.; Schuur, B.; de Haan, A. B. Biobutanol Recovery Using Nonfluorinated Task-Specific Ionic Liquids. Ind. Eng. Chem. Res. 2012, 51, 8293.

(8) Freire, M. G.; Cláudio, A. F. M.; Araujo, J. M. M.; Coutinho, A. P.; Marrucho, I. M.; Lopes, J. N. C.; Rebelo, L. P. N. Aqueous Biphasic
Systems: A Boost Brought about by Using Ionic Liquids. Chem. Soc. Rev. 2012, 41, 4966

(9) Coleman, D.; Gathergood, N. Biodegradation Studies of Ionic Liquids. Chem. Soc. Rev. 2010, 39, 600.

(10) Stolte, S.; Steudte, S.; Areitioaurtena, O.; Pagano, F.; Thöming, J.; Stepnowski, P.; Igartua, A. Ionic Liquids as Lubricants or Lubrication Additives: An Ecotoxicity and Biodegradability Assessment. Chemosphere 2012, 89, 1135.

(11) Hou, X. D.; Liu, Q. P.; Smith, T. J.; Li, N.; Zong, M. H. Evaluation of Toxicity and Biodegradability of Cholinium Amino Acids Ionic Liquids. PLoS One 2013, 8, e59145.

(12) Meck, W. H.; Williams, C. L. Choline Supplementation during Prenatal Development Reduces Proactive Interference in Spatial Memory. Dev. Brain Res. 1999, 118, 51.

(13) Zeisel, S. H.; da Costa, K.-A. Choline: An Essential Nutrient for Public Health Steven. Nutr. Rev. 2009, 67, 615.

(14) Xie, Y.; Xing, H.; Yang, Q.; Bao, Z.; Su, B.; Ren, Q. Aqueous Biphasic System Containing Long Chain Anion-Functionalized Ionic Liquids for High-Performance Extraction. ACS Sustainable Chem. Eng. 2015, 3, 3365.

(15) Shahriari, S.; Tomé, L. C.; Araújo, J. M. M.; Rebelo, L. P. N.; Coutinho, J. A. P.; Marrucho, I. M.; Freire, M. G. Aqueous Biphasic Systems: A Benign Route Using Cholinium-Based Ionic Liquids. RSC Adv. 2013, 3, 1835.

(16) Pereira, J. F. B.; Vicente, F.; Santos-Ebinuma, V. C.; Araújo, J. M.; Pessoa, A.; Freire, M. G.; Coutinho, J. A. P. Extraction of Tetracycline from Fermentation Broth Using Aqueous Two-Phase Systems Composed of Polyethylene Glycol and Cholinium-Based Salts. Process Biochem. 2013, 48, 716.

(17) Torres-Acosta, M. A.; Pereira, J. F. B.; Freire, M. G.; AguilarYáñez, J. M.; Coutinho, J. A. P.; Titchener-Hooker, N. J.; RitoPalomares, M. Economic Evaluation of the Primary Recovery of Tetracycline with Traditional and Novel Aqueous Two-Phase Systems. Sep. Purif. Technol. 2018, 203, 178.

(18) Freire, M. G.; Neves, C. M. S. S.; Canongia Lopes, J. N.; Marrucho, I. M.; Coutinho, J. A. P.; Rebelo, L. P. N. Impact of SelfAggregation on the Formation of Ionic-Liquid-Based Aqueous Biphasic Systems. J. Phys. Chem. B 2012, 116, 7660.

(19) Passos, H.; Trindade, M. P.; Vaz, T. S. M.; da Costa, L. P.; Freire, M. G.; Coutinho, J. A. P. The Impact of Self-Aggregation on the Extraction of Biomolecules in Ionic-Liquid-Based Aqueous Two-Phase Systems. Sep. Purif. Technol. 2013, 108, 174.

(20) Belchior, D. C. V.; Sintra, T. E.; Carvalho, P. J.; Soromenho, M. R. C.; Esperança, J. M. S. S.; Ventura, S. P. M.; Rogers, R. D.; Coutinho, J. A. P.; Freire, M. G. Odd-Even Effect on the Formation of Aqueous Biphasic Systems Formed by 1-Alkyl-3-Methylimidazolium Chloride Ionic Liquids and Salts. J. Chem. Phys. 2018, 148, 193842.

(21) Ventura, S. P. M.; Sousa, S. G.; Serafim, L. S.; Lima, A. S.; Freire, M. G.; Coutinho, J. A. P. Ionic-Liquid-Based Aqueous Biphasic Systems with Controlled pH: The Ionic Liquid Anion Effect. J. Chem. Eng. Data 2012, 57, 507.

(22) Najdanovic-Visak, V.; Lopes, J.; Visak, Z. P.; Trindade, J.; Rebelo, L. P. N. Salting-out in Aqueous Solutions of Ionic Liquids and $\mathrm{K}_{3} \mathrm{PO}_{4}$ : Aqueous Biphasic Systems and Salt Precipitation. Int. J. Mol. Sci. 2007, $8,736$.

(23) Cláudio, A. F. M.; Ferreira, A. M.; Shahriari, S.; Freire, M. G.; Coutinho, J. A. P. Critical Assessment of the Formation of Ionic-LiquidBased Aqueous Two-Phase Systems in Acidic Media. J. Phys. Chem. B 2011, 115, 11145.

(24) Deive, F. J.; Rodríguez, A.; Marrucho, I. M.; Rebelo, L. P. N. Aqueous Biphasic Systems Involving Alkylsulfate-Based Ionic Liquids. J. Chem. Thermodyn. 2011, 43, 1565.

(25) Adamová, G.; Gardas, R. L.; Rebelo, L. P. N.; Robertson, A. J.; Seddon, K. R. Alkyltrioctylphosphonium Chloride Ionic Liquids: Synthesis and Physicochemical Properties. Dalton Trans. 2011, 40, 12750.

(26) Rocha, M. A. A.; Neves, C. M. S. S.; Freire, M. G.; Russina, O.; Triolo, A.; Coutinho, J. A. P.; Santos, L. M. N. B. F. Alkylimidazolium 
Based Ionic Liquids: Impact of Cation Symmetry on Their Nanoscale Structural Organization. J. Phys. Chem. B 2013, 117, 10889.

(27) Rocha, M. A. A.; Coutinho, J. A. P.; Santos, L. M. N. B. F. Cation Symmetry effect on the Volatility of Ionic Liquids. J. Phys. Chem. B 2012, 116, 10922.

(28) Rocha, M. A. A.; Coutinho, J. A. P.; Santos, L. M. N. B. F. Vapor Pressures of 1,3-Dialkylimidazolium Bis(trifluoromethylsulfonyl)imide Ionic Liquids with Long Alkyl Chains. J. Chem. Phys. 2014, 141, 134502.

(29) Quental, M. V.; Caban, M.; Pereira, M. M.; Stepnowski, P.; Coutinho, J. A. P.; Freire, M. G. Enhanced Extraction of Proteins Using Cholinium-Based Ionic Liquids as Phase-Forming Components of Aqueous Biphasic Systems. Biotechnol. J. 2015, 10, 1457.

(30) Merchuk, J. C.; Andrews, B. A.; Asenjo, J. A. Aqueous Two-Phase Systems for Protein Separation. J. Chromatogr., Biomed. Appl. 1998, $711,285$.

(31) Seader, J. D.; Henley, E. J. Separation Process Principles, 2nd ed.; John Wiley \& Sons, Inc.: New York, 2006.

(32) Setschenow, J. Z. Über Die Konstitution Der Salzlösungen Auf Grund Ihres Verhaltens Zu Kohlensäure. Z. Phys. Chem. 1889, 4, 117.

(33) Hey, M. J.; Jackson, D. P.; Yan, H. The Salting-out Effect and Phase Separation in Aqueous Solutions of Electrolytes and Poly(Ethylene Glycol). Polymer 2005, 46, 2567.

(34) Zafarani-Moattar, M. T.; Hamzehzadeh, S. Phase Diagrams for the Aqueous Two-Phase Ternary System Containing the Ionic Liquid 1-Butyl-3-Methylimidazolium Bromide and Tri-Potassium Citrate at T $=(278.15,298.15$, and 318.15) K. J. Chem. Eng. Data 2009, 54, 833.

(35) Zafarani-Moattar, M. T.; Hamzehzadeh, S. Salting-out Effect, Preferential Exclusion, and Phase Separation in Aqueous Solutions of Chaotropic Water-Miscible Ionic Liquids and Kosmotropic Salts: Effects of Temperature, Anions, and Cations. J. Chem. Eng. Data 2010, $55,1598$.

(36) Kurnia, K. A.; Lima, F.; Cláudio, A. F. M.; Coutinho, J. A. P.; Freire, M. G. Hydrogen-Bond Acidity of Ionic Liquids: An Extended Scale. Phys. Chem. Chem. Phys. 2015, 17, 18980.

(37) TURBOMOLE V6.1; Universität Karlsruhe Forschungszentrum Karlsruhe GmbH, 2009; available from http//www.turbomole.com.

(38) Eckert, F.; Klamt, A. COSMOtherm, version C2.1, release 01.08; COMsol GmbH \& Co. KG: Leverkusen, Germany, 2006.

(39) Araújo, J. M. M.; Ferreira, R.; Marrucho, I. M.; Rebelo, L. P. N. Solvation of Nucleobases in 1,3-Dialkylimidazolium Acetate Ionic Liquids: NMR Spectroscopy Insights into the Dissolution Mechanism. J. Phys. Chem. B 2011, 115, 10739.

(40) Costa, A. J. L.; Soromenho, M. R. C.; Shimizu, K.; Marrucho, I. M.; Esperança, J. M. S. S.; Lopes, J. N. C.; Rebelo, L. P. N. Liquid-Liquid Equilibrium of Cholinium-Derived Bistriflimide Ionic Liquids with Water and Octanol. J. Phys. Chem. B 2012, 116, 9186.

(41) de Ferro, A. M.; Reis, P. M.; Soromenho, M. R. C.; Bernardes, C. E. S.; Shimizu, K.; Freitas, A. A.; Esperança, J. M. S. S.; Canongia Lopes, J. N.; Rebelo, L. P. N. Designing the ammonium cation to achieve a higher hydrophilicity of bistriflimide-based ionic liquids. Phys. Chem. Chem. Phys. 2018, 20, 19307.

(42) Rengstl, D.; Kraus, B.; Van Vorst, M.; Elliott, G. D.; Kunz, W. Effect of choline carboxylate ionic liquids on biological membranes. Colloids Surf., B 2014, 123, 575.

(43) Freire, M. G.; Carvalho, P. J.; Silva, A. M. S.; Santos, L. M. N. B. F.; Rebelo, L. P. N.; Marrucho, I. M.; Coutinho, J. A. P. Ion Specific Effects on the Mutual Solubilities of Water and Hydrophobic Ionic Liquids Ion Specific Effects on the Mutual Solubilities of Water and Hydrophobic Ionic Liquids. J. Phys. Chem. B 2009, 113, 202.

(44) Zafarani-Moattar, M. T.; Hamzehzadeh, S. Partitioning of Amino Acids in the Aqueous Biphasic System Containing the Water-Miscible Ionic Liquid 1-Butyl-3-Methylimidazolium Bromide and the WaterStructuring Salt Potassium Citrate. Biotechnol. Prog. 2011, 27, 986.

(45) Chemspider, The free chemical database, http:// www.chemspider. com. 